\title{
The Importance of the DEIC/GEMIC/SBC 2021 Position Statement on Diagnosis and Treatment of Cardiac Amyloidosis to Clinical Practice
}

\author{
Marcus Vinicius Simões ${ }^{10}$ and Fabio Fernandes ${ }^{2}$ \\ Divisão de Cardiologia, Departamento de Clínica Médica da Faculdade de Medicina de Ribeirão Preto da Universidade de São Paulo, ${ }^{1}$ \\ Ribeirão Preto, SP - Brazil \\ Instituto do Coração - HC FMUSP,2 São Paulo, SP - Brazil
}

The latest edition of Arquivos Brasileiros de Cardiologia has brought us the publication of the Position Statement on Diagnosis and Treatment of Cardiac Amyloidosis by the DEIC/ GEMIC/SBC. This document was the result of the collaborative efforts of many experts in the area, exponents of cardiology in Brazil, but it also included other specialists, such as neurologists, hematologists and nuclear physicians.

The need to bring together this group of collaborators reflects a fundamental aspect of amyloidosis, namely, a complex and multifaceted disease that affects multiple organs and requires the assistance of several specialties for comprehensive management. This was, in fact, a positive aspect of the document: its scope and multidisciplinary character.

It is here appropriate to state the reasons that led the DEIC/GEMIC/SBC to produce this document, fulfilling the mission of producing documents that contribute to updating science and medical education, offering the signposts that are necessary to guide the practice of cardiology based on scientific evidence. The sense of urgency for this action was the clear perception of the recent and profound reformulation of concepts regarding cardiac amyloidosis (CA), including evidence that it is a more prevalent disease than previously imagined, the significant advance in imaging methods that has made non-invasive diagnosis possible, and the emergence of new specific treatments that can change the natural history of the disease (Figure 1).

\section{Cardiac amyloidosis is a frequent cause of heart failure with preserved ejection fraction}

The main cardiological phenotype of CA is that of an infiltrative restrictive cardiomyopathy, whose more general aspects of symptoms, increased thickness of the ventricular walls, and concentric remodeling of the left ventricle, overlap with those of heart failure with preserved ejection fraction (HFpEF), produced by risk factors such as high blood pressure, obesity, diabetes, and aging. Thus, CA should be considered as a differential diagnosis in patients who exhibit HFpEF.

\section{Keywords}

Amyloidosis; Cardiomyopathy; Diagnostic Methods; Treatment.

Mailing Address: Marcus Vinicius Simões •

Av. Bandeirantes 3900, Campus da USP, Bairro Monte Alegre,

Ribeirão Preto - SP. Postal Code:14049-900

E-mail:msimoes@fmrp.usp.br

Manuscript received September 09, 2021, revised manuscript September 24, 2021, accepted September 24, 2021.

DOI: https://doi.org/10.36660/abchf.20210021
It is worth remembering that, among the different amyloidogenic proteins that can be deposited in the myocardium and cause CA, the wild type transthyretin form (ATTR-CAwt), which is closely linked to aging, is the most frequently reported in several series of specialized medical literature. This has been repeated both in large reference centers and in real world registers. ${ }^{2}$ Accordingly, a recent article has shown a very significant increase in CA as a cause of hospitalizations due to heart failure in several regions of the United States, between 2000 and 2012, exceeding the rate of 65 cases per 100,000 people/year, indicating that $\mathrm{CA}$ is not a rare disease according to the current criteria of the World Health Organization (Figure 2). ${ }^{3}$

It is important to remember that there are no similar statistics in our country to confirm the higher prevalence of CA in the Brazilian population. This is a gap that we hope will be filled in the near future.

\section{Non-invasive diagnosis using bone tracer scintigraphy}

Of all the advances in the clinical scenario of CA, the one with the greatest impact has been the development of the bases for non-invasive diagnosis of ATTR-CA, dispensing the use of endomyocardial biopsy. In the celebrated study published by Perugini et al. in 2005, it was well demonstrated that patients with ATTR-CA exhibited intense and anomalous myocardial accumulation of some bisphosphonate agents used for bone scintigraphy. In this seminal study, only patients with ATTR-CA exhibited ${ }^{99 m}$ Tc-DPD (3,3-diphosphono-1,2propanedicarboxylic acid) uptake equivalent or superior to costal arch uptake (grades 2 or 3), while none of the patients with AC linked to light chains (AL form) showed the same behavior. $^{4}$

It was subsequently identified that a certain percentage ( $20 \%$ to $30 \%$ ) of patients with AL-CA could also exhibit expressive uptake of bone markers, making it essential to rule out the presence of immunoglobulin light chains for diagnostic confirmation of ATTR-CA in patients with positive cardiac scintigraphy using bone markers. In the fundamental study by Gillmore et al., this set of results (cardiac scintigraphy with grade 2 or 3 bone marker uptake + light chain exclusion) had $100 \%$ specificity and predictive value, thus defining the noninvasive criteria for diagnosis of ATTR-CA, which have gone on to become a standard of conduct worldwide. ${ }^{5}$

These fundamental aspects have guided the choices made by the panel of experts who created the flowchart for the diagnosis of CA in our Position Statement, which is reproduced in Figure 3. This alone is the most important item of the entire document, and we hope that it will be able to guide the diagnostic flow of $\mathrm{CA}$ in routine cardiology practice. 


\section{Editorial}

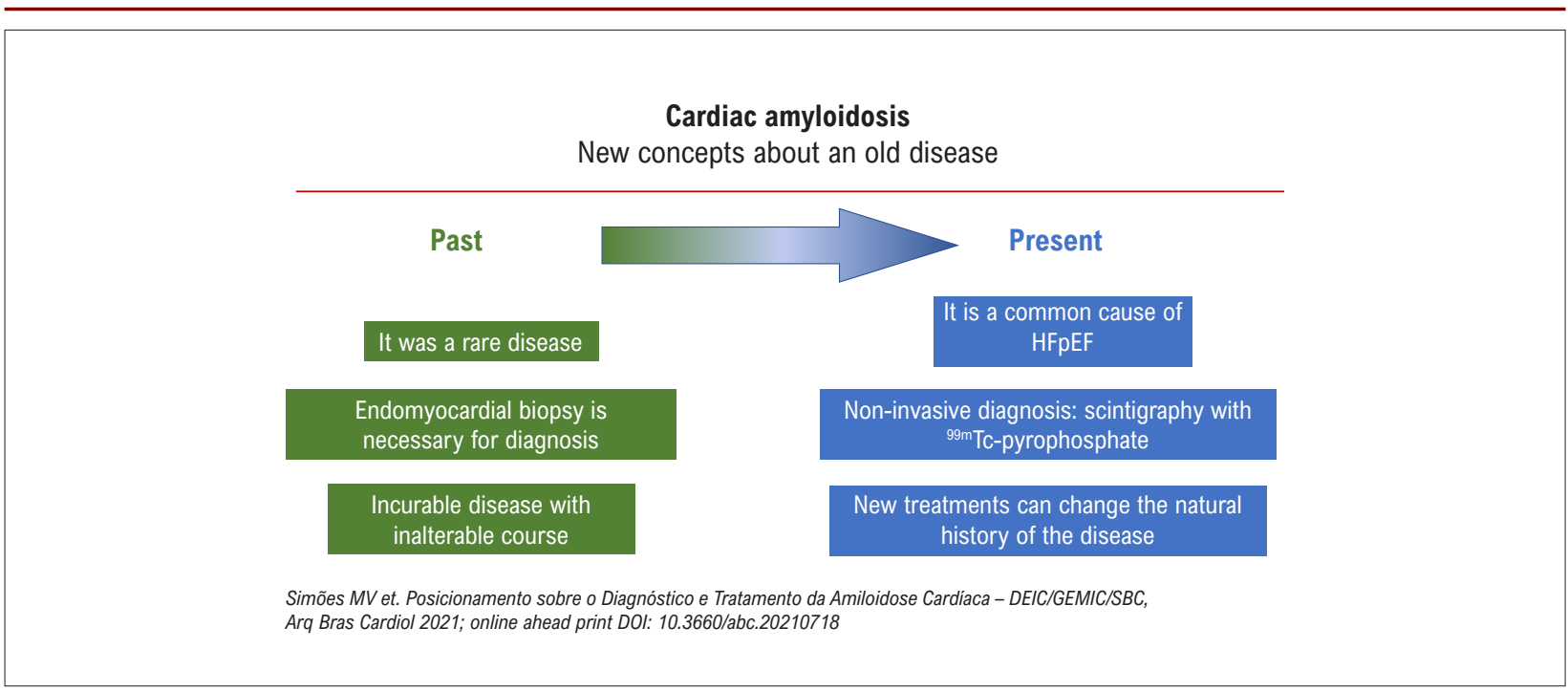

Figure 1 - Illustration outlining the aspects involved in the profound reformulation of concepts regarding cardiac amyloidosis that have expanded in recent years. HFpEF: heart failure with preserved ejection fraction.

\section{Increase of HF hospitalizations associated to Cardiac amyloidosis in US}

Gilstrap LG et al. Circ Heart Fail 2019;12:e005407

A Prevalence of Cardiac Amyloidosis in the United States in 2000 and 2012

2000

2012

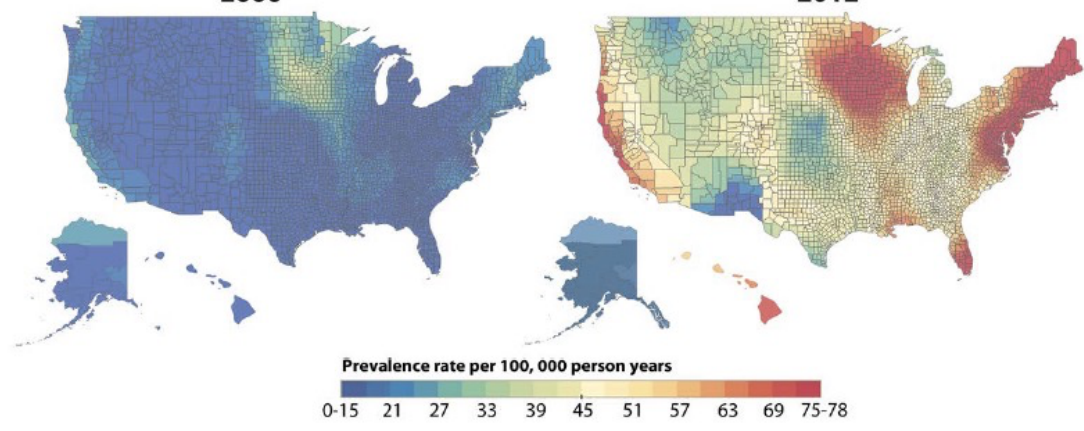

Figure 2 - Heat map showing the increased prevalence of cardiac amyloidosis as a cause associated with hospitalizations due to heart failure in the United States, between 2000 and 2012. Reproduced from (Gilstrap et al., 2019).

\section{New specific treatments}

There is no doubt that any efforts made to improve our ability to diagnose a given condition are only fully valid and compensated when we are able link this finding to the use of specific treatments capable of alleviating symptoms and prolonging our patients' lives. The emergence of new therapies specific to CA has been the fundamental spring that has fueled research and initiatives to raise the level of care for more accurate and earlier diagnosis of CA.

The transthyretin tetramer stabilizer tafamidis was the first drug for specific treatment of ATTR-CA to be tested in a multicenter, randomized, blinded study, the ATTRACT study. ${ }^{6}$ The main results of ATTRACT have shown that the use of tafamidis was associated with a significant reduction in all-cause mortality by $30 \%(\mathrm{HR}=0.70,95 \%$ $\mathrm{Cl}: 0.51$ to 0.96 ) and a reduction in hospitalizations/year of around $32 \%(\mathrm{HR}=0.68 \% ; 95 \% \mathrm{Cl}: 0.56$ to 0.81$)$. In this manner, tafamidis is the first specific treatment for ATTR-CA capable of changing the natural history of the disease, and it has received approval from the Brazilian Health Regulatory Agency for the treatment of ATTR-CA in Brazil.

Other very promising molecules are currently being developed and tested in phase 3 multicenter trials, including 


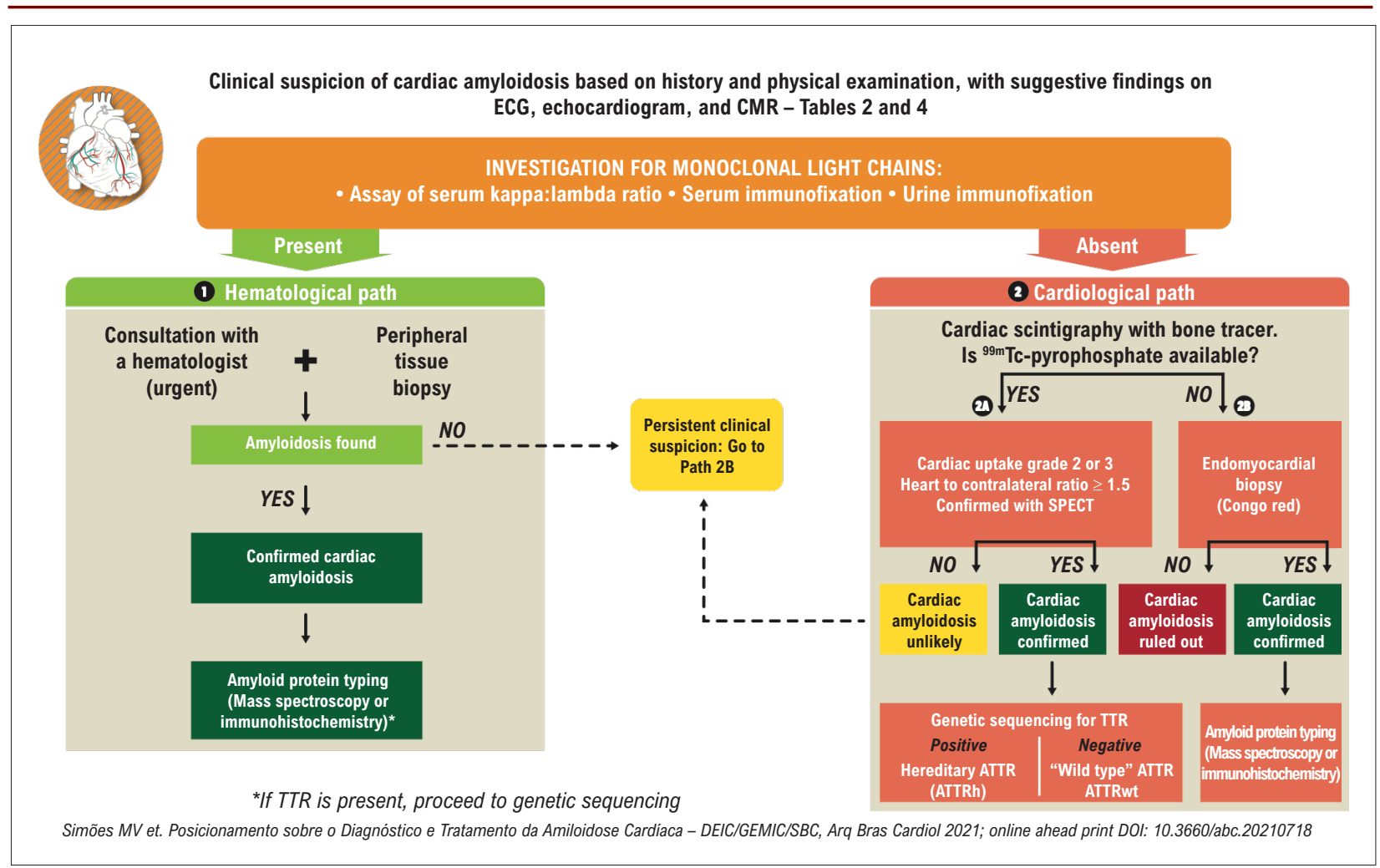

Figure 3 - Flowchart for diagnosis of $C A$

strategies for silencing the gene expression of TTR production through "small interference RNA" (patisiran) and "anti-sense" oligonucleotide (eplontersen) platforms.

It is important to remember that we have recently witnessed the incorporation of significant advances in the treatment of AL-CA, using monoclonal antibodies and autologous bone marrow transplantation, which are associated with a surprising positive impact on mortality due to AL-CA, provided that patients are referred during earlier stages of disease evolution, which highlights the need for more accurate and quicker diagnosis of this disease. ${ }^{7}$

\section{Final comments}

As reviewed here, there is strong evidence to suggest that CA is a relatively prevalent but broadly underdiagnosed condition. The emergence of new therapies capable of changing the natural history of this serious disease has brought about the need for earlier and safer diagnosis. The publication of the DEIC/GEMIC/SBC 2021 Position Statement on Diagnosis and Treatment of Cardiac Amyloidosis takes on fundamental importance at this moment by contributing to the incorporation of these new concepts in clinical practice and providing signposts based on the most recent scientific evidence.

\section{Author Contributions}

Conception and design of the research; Acquisition of data; Analysis and interpretation of the data; Statistical analysis; Obtaining financing; Writing of the manuscript; Critical revision of the manuscript for intellectual content: Simões $\mathrm{MV}$, Fernandes F.

\section{Potential Conflict of Interest}

No potential conflict of interest relevant to this article was reported.

\section{Sources of Funding}

There were no external funding sources for this study.

\section{Study Association}

This study is not associated with any thesis or dissertation work.

\section{Ethics approval and consent to participate}

This article does not contain any studies with human participants or animals performed by any of the authors. 


\section{Editorial}

\section{References}

1. Rapezzi C, Lorenzini M, Longhi S, Milandri A, Gagliardi C, Bartolomei I, et al. Cardiac Amyloidosis: The Great Pretender. Heart Fail Rev. 2015;20(2):11724. doi: 10.1007/s10741-015-9480-0.

2. Lane T, Fontana M, Martinez-Naharro A, Quarta CC, Whelan CJ, Petrie A, et al. Natural History, Quality of Life, and Outcome in Cardiac Transthyretin Amyloidosis. Circulation. 2019;140(1):16-26. doi: 10.1161/ CIRCULATIONAHA.118.038169.

3. Gilstrap LG, Dominici F, Wang Y, El-Sady MS, Singh A, Di Carli MF, et al. Epidemiology of Cardiac Amyloidosis-Associated Heart Failure Hospitalizations Among Fee-for-Service Medicare Beneficiaries in the United States. Circ Heart Fail. 2019;12(6):e005407. doi: 10.1161/CIRCHEARTFAILURE.118.005407.

4. Perugini E, Guidalotti PL, Salvi F, Cooke RM, Pettinato C, Riva L, et al. Noninvasive Etiologic Diagnosis of Cardiac Amyloidosis using 99mTc-
3,3-Diphosphono-1,2-Propanodicarboxylic Acid Scintigraphy. J Am Coll Cardiol. 2005;46(6):1076-84. doi: 10.1016/j.jacc.2005.05.073.

5. Gillmore JD, Maurer MS, Falk RH, Merlini G, Damy T, Dispenzieri A, et al. Nonbiopsy Diagnosis of Cardiac Transthyretin Amyloidosis. Circulation. 2016;133(24):2404-12. doi: 10.1161/ CIRCULATIONAHA.116.021612.

6. MaurerMS, SchwartzJH, Gundapaneni B, Elliott PM, Merlini G, WaddingtonCruz M, et al. Tafamidis Treatment for Patients with Transthyretin Amyloid Cardiomyopathy. N Engl J Med. 2018;379(11):1007-16. doi: 10.1056/ NEJMoa1805689.

7. Gertz MA, Dispenzieri A. Systemic Amyloidosis Recognition, Prognosis, and Therapy: A Systematic Review. JAMA. 2020;324(1):79-89. doi: 10.1001/ jama.2020.5493. 